\title{
Vaccination of blood donors against hepatitis $B$ is feasible, safe and cost-effective
}

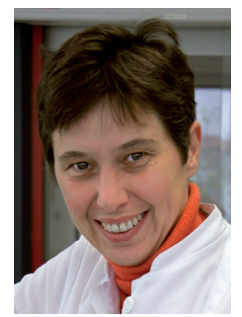

\author{
"Taken together, in theory, HBV vaccination offers \\ the near elimination of transfusion infections while \\ representing a potential cost reduction."
}

\section{Barbara C Gärtner}

Institute for Microbiology \& Hygiene, University of the Saarland, D-66421 Homburg/Saar, Germany n. Tel.: +49 68411623900 = Fax: +49684 11623985 = barbara.gaertner@uks.eu

Hepatitis B virus (HBV) is an important cause of post-transfusion hepatitis. For many years different strategies have been implemented to reduce the number of transfusion-transmitted infections from blood donations. Selection of donors and testing of blood products play a major role in the prevention of blood-borne infections. Current regulations for preventing $\mathrm{HBV}$ transmission stipulate testing for hepatitis B surface antigen (HBsAg) and antibodies specific to hepatitis B core antigen (anti-HBc) in many countries, such as in Germany. However, during the early phase of infection an antibody response is not yet apparent and insufficient antigen might be present to allow detection, resulting in a diagnostic window of approximately 50 days during which virus is present but is essentially invisible to current testing procedures [1].

For other blood-borne infections like HCV and HIV the mandatory tests also include nucleic acid techniques (NATs). The use of NATs has been proposed to reduce the current risk of $\mathrm{HBV}$ transfusion infection (one in 250,000 in Germany) [2,3], but is also subject to restriction due to a diagnostic window, and even when using a combination of $\mathrm{HBsAg}$, anti-HBc and NATs, the residual risk of $\mathrm{HBV}$ transmission (one in 880,000) remains clearly higher than those of HIV (one in 4,640,000) or HCV (one in $2,730,000)[1,4,5]$.

\section{HBV assays for screening of blood donations}

Historically, $\mathrm{HBsAg}$ was the first mandatory HBV test in transfusion medicine. Testing for $\mathrm{HBsAg}$ alone is limited by the fact that HBsAg mutants might not be detected by some assays and, more importantly, low-level $\mathrm{HBsAg}$ replication in chronic infections might be missed owing to the detection limit of the assays. Adding anti-HBc to the test panel enables the detection of most of these samples, leaving the initial window period as the major risk of $\mathrm{HBV}$ transmission. One of the major drawbacks of anti-HBc inclusion is this lack of a confirmation test.

A repeat donor with a first-time-positive anti-HBc assay is suspected of HBV infection with possibly infectious donations during a window period prior to seroconversion, triggering a look-back procedure. This includes contacting of all recipients of blood products donated during a defined period prior to seroconversion. All recipients have to be tested for $\mathrm{HBV}$ infection, resulting in a significant psychological stress and high costs.

Although in the case of a true seroconversion this approach is important to find recipients at risk, the majority of seroconversions are not a result of a HBV infection but of unspecific or false-positive test results. Even using another lot of a given assay might result in discrepancies with samples reacting positive at a low level. As a consequence, a virtual seroconversion is detected and a look-back has to be initiated. This is essentially the same as for HBsAg. However, a seroconversion in HBsAg could be excluded or confirmed by other assays such as a neutralization assay. Moreover, infectivity could be proven or excluded by NATs. Thus, false positive screening results generally do not result in a look-back. In contrast with the HBsAg situation, which has different confirmation methods available, a seroconversion detected using anti-HBc could not be confirmed by any other assays, resulting in a significant number of unnecessary look-back procedures without underlying infection of the donor.

At present, HBV NATs are not mandatory in many countries; however, some institutions voluntarily test for HBV DNA and some commercially available multiplex NATs include HIV and HCV

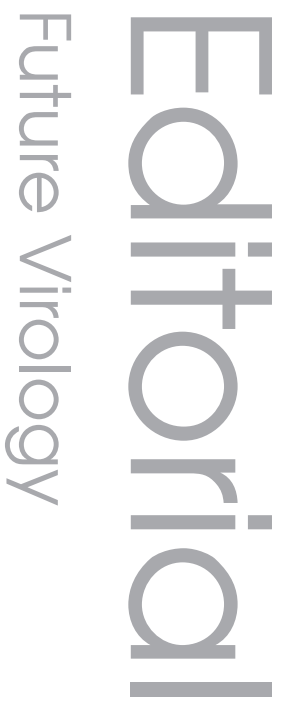

\section{Keywords}

- anti-HBC $=$ apheresis donor

- blood donation $=\mathrm{HBsAg}$

- health economics = hepatitis

- PCR vaccination

- repeat donor - transfusion

- whole blood donor

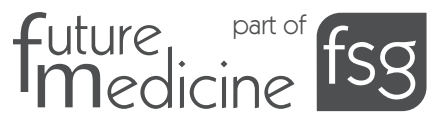


or other viruses next to HBV. HBV NATs clearly reduce the number of HBV transmissions; however. a window period still has to be considered and, moreover, NATs do not solve the problem of false-positive anti-HBc seroconversions.

Taken together, an alternative method for increasing the safety of blood donations seems to be absolutely essential, and if possible, without relying on anti-HBc testing.

\section{Vaccination as a tool to increase safety \& decrease costs}

An alternative approach to all these test strategies may be to vaccinate blood donors against HBV. The HBV vaccine has been on the market for many years and has proven its safety and efficacy. Successful vaccination would essentially eliminate infection and transmission, but may also require significant changes to current testing regulations.

In a cost-benefit analysis we calculated different models for the vaccination of blood donors and compared the costs and benefits with various test strategies. Vaccinating donors with a yearly anti-Hbs test to prove immunity, including a booster dose in the case of a low titer $(<100 \mathrm{IU} / \mathrm{l})$, without additional testing of blood products would result in a reduction of cost of $14 \%$ within a period of 20 years [6]. Thus, vaccination would not increase but instead decrease costs. However, owing to safety reasons one might argue that testing could not be completely abolished. In a model with vaccination added to the mandatory testing, the absolute cost per prevented infection would be approximately 2 million. This is roughly the same as for the prevention of $\mathrm{HCV}$ transmission by HCV NATs in Germany [6].

\section{6}

...donation testing might be safely

omitted if an anti-Hbs titer of at least $100 \mathrm{IU} / \mathrm{I}$ is secured in the donors."

A vaccination strategy without testing of blood donations harbors a residual risk of HBV transmission. Stramer and coworkers showed clearly that individuals might be infected under certain circumstances despite HBV vaccination [7]. Characteristically, these donors were infected with HBV genotypes (C2, F1, B2) different to the vaccine genotype (A2) in combination with a low anti-Hbs titer $(<100 \mathrm{IU} / \mathrm{l})$. It is tempting to speculate that higher anti-Hbs titers are necessary to avoid infections with nonvaccine genotypes. Moreover, it is unclear if donations of vaccinated donors with anti-Hbs titers are infectious at all. A study by Satake and coworkers indicated that donations with detectable levels of
HBV DNA in combination with anti-HBs were not infectious in any of 22 recipients as compared with $27 \%$ among 37 recipients with HBV DNA but without anti-Hbs [8]. Other studies underline these results [9-11].

Thus, donation testing might be safely omitted if an anti-Hbs titer of at least $100 \mathrm{IU} / \mathrm{l}$ is secured in the donors.

"In our interview-based study, $97 \%$ of
donors indicated they would agree to
vaccination, making the prospect of
vaccination strategies realistic."

A general problem in $\mathrm{HBV}$ testing and vaccination are viruses with mutations within the HBsAg region [11]. Some of them are not detectable in the HBsAg assays, while others might represent a vaccine escape, thus even vaccinated individuals with high antibody titers might not be immune to these mutants. Fortunately, these vaccine escape mutants mainly occur in HBVinfected patients after passive vaccination and are only rarely found in a donor population [12]. HBV NATs seem to be the most suitable tests to detect these mutants. In individuals infected with escape mutants, even anti-HBc seems not to be a reliable marker since quite a few have been shown to react negatively [11]. These mutants comprise a risk that could not be eliminated by vaccination, not even in combination with high anti-Hbs titers and constitute the residual risk of a vaccination strategy without donation testing. However this risk should be extremely low.

Taken together, in theory, $\mathrm{HBV}$ vaccination offers the near elimination of transfusion infections while representing a potential cost reduction.

\section{From theory to the real world}

Introducing vaccination for blood donors presents some challenges:

- From a technical perspective the willingness of donors to be vaccinated is an absolute prerequisite. In our interview-based study, 97\% of donors indicated they would agree to vaccination, making the prospect of vaccination strategies realistic [6]. Moreover, vaccination results in a different management of donations from immune donors (without donation testing) and from nonimmune donors (with donation testing) owing to donations of nonresponders or donors without completed vaccination.

- Ethically and politically, the vaccination of donors for mainly altruistic reasons has to be carefully considered. The intention to vaccinate 
focuses on the safety of a blood product and not the health of the vaccinee. The benefits for the vaccinee have to be carefully balanced against the very rare potentially severe side effects (e.g. allergic reactions) under this nontypical altruistic vaccine indication.

At present, a change to a vaccination-based strategy would also require a change to the transfusion regulations. Current regulations in Germany allow for altruistic vaccination of donors only where no alternative source for the product is available. Since the potential advantages from such a change include the near elimination of transfusion transmission of $\mathrm{HBV}$ along with an overall reduction in costs from prevention measures, political support may be expected.

\section{Bibliography}

Papers of special note have been highlighted as:

- of interest

-. of considerable interest

1. Offergeld R, Faensen D, Ritter S, Hamouda O. Human immunodeficiency virus, hepatitis $\mathrm{C}$ and hepatitis $\mathrm{B}$ infections among blood donors in Germany 20002002: risk of virus transmission and the impact of nucleic acid amplification testing. Eurosurveillance 10(2), 8-11 (2005).

2. Fang CT. Blood screening for HBV DNA. J. Clin Virol. 36(Suppl. 1), S30-S32 (2006)

3. Lelie N, Heaton A. Hepatitis B A review of the role of NAT in enhancing blood safety. J. Clin. Virol. 36(Suppl. 1), S1-S2 (2006).

4. Dodd RY, Notari EP, Stramer SL. Current prevalence and incidence of infectious disease markers and estimated window-period risk in the American Red Cross blood donor population. Transfusion 42(8), 975-979 (2002).

5. Schreiber GB, Busch MP, Kleinman SH, Korelitz JJ. The risk of transfusiontransmitted viral infections. The retrovirus epidemiology donor study. N. Engl. J. Med. 334(26), 1685-1690 (1996).

Taken together, the vaccination of blood donors is feasible and increases safety at reduced or at least acceptable costs. At present political support is the prerequisite to implement standard $\mathrm{HBV}$ vaccination as a new tool to reduce transfusion transmitted infections within the next years.

\section{Financial \& competing interests disclosure \\ $B C$ Gärtner received lecture fees from and has had a study partly financed by GlaxoSmithKline. The author has no other relevant affiliations or financial involve- ment with any organization or entity with a financial interest in or financial conflict with the subject matter or materials discussed in the manuscript apart from those disclosed. \\ No writing assistance was utilized in the production of this manuscript.}

6. Fischinger JM, Stephan B, Wasserscheid K, Eichler H, Gärtner BC. A cost-benefit analysis of blood donor vaccination as an alternative to additional DNA testing for reducing transfusion transmission of hepatitis B virus. Vaccine 28(49), 7797-7802 (2010).

- Cost-benefit analysis of different vaccination and testing strategies showing that vaccination is feasible and cost-effective.

7. Stramer SL, Wend U, Candotti D et al. Nucleic acid testing to detect HBV infection in blood donors. N. Engl. J. Med. 364(3), 236-247 (2011).

- Outstanding study showing that vaccinated donors could be infected with hepatitis $B$ virus under certain circumstances.

8. Satake M, Taira R, Yugi $\mathrm{H}$ et al. Infectivity of blood components with low Hepatitis B virus DNA levels identified in a lookback program. Transfusion 47(7), 1197-1205 (2007).
- Important study showing that hepatitis B virus-positive donors with hepatitis $B$ antigen antibodies present in the blood product did not transmit infection.

9. Prince AM, Lee DH, Brotman B. Infectivity of blood from PCR-positive, $\mathrm{HBsAg}$ negative, anti-HBs-positive cases of resolved Hepatitis B infection. Transfusion 41, 329-332 (2001).

10. Dreier J, Kröger M, Diekmann J, Götting C, Kleesiek K. Low-level viraemia of Hepatitis B virus in an anti-HBc-and anti-Hbs-positive blood donor Transfus. Med. 14, 97-103 (2004)

11. Gerlich WH. Breakthrough of Hepatitis B virus escape mutants after vaccination and virus reactivation. J. Clin. Virol. 36(Suppl. 1), S18-S22 (2006)

12. Chang $\mathrm{MH}$. Breakthrough $\mathrm{HBV}$ infection in vaccinated children in Taiwan: surveillance for HBV mutants. Antivir. Ther. 15 (3 Pt B), 463-469 (2010). 